\title{
Kernos
}

Revue internationale et pluridisciplinaire de religion grecque antique

1 | 1988

Varia

\section{Présentation du programme Mentor. Bibliographie critique informatisée de la religion grecque antique}

\section{Vinciane Pirenne-Delforge}

\section{(2) OpenEdition}

\section{Journals}

\section{Édition électronique}

URL : http://journals.openedition.org/kernos/157

DOI : $10.4000 /$ kernos. 157

ISSN : 2034-7871

\section{Éditeur}

Centre international d'étude de la religion grecque antique

\section{Édition imprimée}

Date de publication : 1 janvier 1988

ISSN : 0776-3824

\section{Référence électronique}

Vinciane Pirenne-Delforge, «Présentation du programme Mentor. Bibliographie critique informatisée de la religion grecque antique », Kernos [En ligne], 1 | 1988, mis en ligne le 01 février 2011, consulté le 26 avril 2019. URL : http://journals.openedition.org/kernos/157 


\title{
PRÉSENTATION DU PROGRAMME MENTOR. BIBLIOGRAPHIE CRITIQUE INFORMATISÉE DE LA RELIGION GRECQUE ANTIQUE
}

\author{
par Vinciane PIRENNE-DELFORGE
}

Il n'est pas nécessaire de justifier longuement la mise en oeuvre du programme Mentor. La création du C.E.R.G.A. et, parallèlement, de la revue Kernos témoignent suffisamment du dynamisme et de l'actualité des recherches relatives à la religion grecque antique. Or il n'existe à ce jour aucun instrument qui permette au chercheur de s'orienter efficacement dans l'abondance des publications sur le sujet. L'Année philologique reste, certes, un outil irremplaçable; mais l'information bibliographique n'y est pas facilement accessible : à côté de la rubrique consacrée en propre à la religion grecque, il en est beaucoup d'autres qui comportent aussi des références intéressant le domaine. D'autre part, il s'agit d'une bibliographie courante qui, par définition, n'offre pas de panorama rétrospectif complet des recherches dans le domaine. Enfin, aucune information critique n'est fournie au lecteur.

C'est à la faveur de leur collaboration au Dictionnaire des religions, pour la partie grecque ${ }^{1}$, que le professeur André Motte et une équipe d'hellénistes liégeois ont particulièrement éprouvé le besoin d'un instrument adéquat. Encore fallait-il le créer.

\section{Les moyens}

L'institution d'accueil est le Centre d'Histoire des Religions de l'Universicé de Liège. Un important subside du Fonds de la Recherche Fondamentale et Collective a permis d'engager un chercheur à temps plein pendant trois années et de faire face aux différentes dépenses de fonctionnement. Par ailleurs, deux crédits du

1 Dictionnaire des religions, Paris, P.U.F., 1984, sous la direction de P. POUPARD (2e éd., 1985; 3e éd. en préparation). 
Fonds National de la Recherche Scientifique ont rendu possible l'acquisition de l'équipement informatique nécessaire. D'autre part, le Centre d'Informatique de Philosophie et Lettres de l'Université a offert au programme son expérience en matière de logiciels de banque de données et a adapté ceux-ci aux besoins spécifiques. L'informatique à la demande, en quelque sorte... ${ }^{2}$. Enfin, Mentor vient de recevoir le soutien de la Société Archéologique d'Athènes et du C.E.R.G.A.

\section{Les objectifs}

1) Matière traitée

Les différents aspects de la religion grecque seront couverts : ouvrages de référence, vocabulaire, mythes et croyances, sentiment religieux, cultes et rites, aspects politiques, juridiques, militaires, sociaux, économiques, rapports avec les arts, la littérature et la philosophie. Mais Mentor entend dépasser le cadre de la religion grecque au sens strict pour s'intéresser aux religions en contact dans la mesure où elles ont influencé la religion grecque et ont été influencées par elle, et à la survivance de certains rites, mythes et symboles à travers les siècles jusqu'au nôtre ('cinéma, thêâtre, roman, etc.).

\section{2) Deux instruments de travail}

Il s'est rapidement avéré que l'abondance des données à traiter ne pourrait être maîtrisée qu'au moyen de l'informatique. La création d'une banque de données est dès lors apparue comme une solution optimale; la possibilité de mises à jour constantes, la souplesse d'interrogation sont des avantages incomparables pour une entreprise bibliographique de ce genre. Fallait-il pour autant renoncer à tout support livresque ? On a pensé qu'un guide bibliographique gardait

2 Pour de plus amples informations sur les aspects informatiques de l'entreprise, voir S. PASLEAU, Cl. PURNELLE-SIMART, G. PURNELLE, Bibliographies informatisées au Centre Informatique de Philosophie et Lettres, in Revue. Informatique et Statistique dans les Sciences humaines, 21(1985), p. 205-226 (p. 218-219 pour le programme Mentor); A. MOTTE, V. PIRENNE, P. WATHELET, L'informatique au service d'une bibliographie critique de la religion grecque. Le programme Mentor entrepris au C.I.P.L., in Revue, 22(1986), p. 93-97. 
toute son utilité et pourrait bénéficier lui aussi des ressources de l'informatique.

Le guide bibliographique, en effet, sera entièrement réalisé à partir de la banque de données. Ces deux instruments seront complémentaires. Le guide offrira un panorama aisément accessible des principales recherches en matière de religion grecque depuis le siècle dernier jusqu'en 1985. Il sera sélectif, surtout pour les publications anciennes. La banque sera beaucoup plus complète et ses mises à jour fourniront la matière des suppléments prévus pour le guide.

3) Structuration de la documentation

Le guide comprendra trois parties :

- le Corpus comportera, dans l'ordre alphabétique des noms d'auteurs, la liste des publications sélectionnées, accompagnées de leur notice critique et d'un numéro d'ordre permettant les renvois. Deux instruments de consultation, forgés selon des méthodes distinctes, faciliteront l'exploitation de cette documentation :

- l'Organon offrira, dans une succession de rubriques thématiques, préalablement établies et hiérarchisées selon un système décimal, un panorama structuré des différents aspects de la religion grecque. Chaque rubrique renverra aux publications reprises dans le Corpus grâce au numéro qui leur est attribué;

- le Thesaurus consistera en un index alphabétique des realia et des notions particulières que l'Organon, en raison de son caractère général, n'aura pu intégrer. Il restera ouvert pendant toute la durée du travail et pourra comporter, pour les entrées les plus importantes, des subdivisions. Il renverra au Corpus de la même manière que l'Organon et comprendra également des renvois internes.

La banque de données sera organisée selon une structuration analogue. On pourra l'interroger soit à partir des champs bibliographiques (auteur, date, lieu d'édition, collection, etc.), soit à partir des codes de rubriques de l'Organon, soit à partir des mots-clés du Thesaurus, soit en cumulant ces données dans des combinaisons particulières. S'ajoutera, en outre, un code chronologique. 
4) Délais de réalisation

La première phase du programme a débuté en octobre 1986. Elle concernait les publications des années 1945-1985 et s'achèvera dans le courant de l'année 1988. Près de 5000 publications ont été recensées tandis que se poursuivait, parallèlement, le dépouillement des publications antérieures.

On peut donc prévoir la mise en service de la banque de données pour le début de l'année 1989 et la parution du guide pour la fin de la même année ou le début de la suivante.

\section{La collaboration}

Un des atouts majeurs du programme est le nombre et la qualité des collaborateurs qu'il a pu réunir. Chacun d'entre eux s'est vu assigner, dans la mesure du possible, un domaine relevant de sa compétence directe. Au résumé des publications, les auteurs sont invités à joindre des éléments d'appréciation, exempts toutefois de polémique. En plus de cette tâche, il leur est demandé de sélectionner les publications qui mérient de figurer dans le guide bibliographique, étant entendu que les ouvrages et articles écartés seront néanmoins repris dans la banque de données. Les critères d'un avis positif sont la valeur scientifique, l'ampleur et l'intérêt de la matière traitée, l'originalité de la méthode appliquée, l'importance du résultat acquis. L'équipe de direction revoit systématiquement tous les formulaires complétés afin de veiller à l'uniformité du travail.

Mentor a pu compter, jusqu'ici, sur quelque cent vingt chercheurs, issus de quinze pays et d'une trentaine d'institutions différentes. Mais l'entreprise se poursuit au-delà de l'année 1985 et de nouveaux collaborateurs seront toujours les bienvenus...

Directeurs du programme : André MOTTE - Paul WATHELET Secrétaire scientifique : Vinciane PIRENNE-DELFORGE.

Adresse : $\quad$ Université de Liège

Programme Mentor

Place du XX-Août 32,

B - 4000 LIEGE

Téléphone : 041/42.00.80. (ext. 568). 